\title{
Enhanced long-lived dark photon signals at the LHC
}

\author{
Mingxuan Du, ${ }^{a}$ Zuowei Liu ${ }^{a, b, c, d}$ and Van Que $\operatorname{Tran}^{a}$ \\ ${ }^{a}$ Department of Physics, Nanjing University, \\ Nanjing 210093, China \\ ${ }^{b}$ Center for High Energy Physics, Peking University, \\ Beijing 100871, China \\ ${ }^{c}$ Nanjing Proton Source Research and Design Center, \\ Nanjing 210093, China \\ ${ }^{d}$ CAS Center for Excellence in Particle Physics, \\ Beijing 100049, China \\ E-mail: mg1722004@smail.nju.edu.cn, zuoweiliu@nju.edu.cn, \\ vqtran@nju.edu.cn
}

ABSTRACT: We construct a model in which the standard model is extended by a hidden sector with two gauge $\mathrm{U}(1)$ bosons. A Dirac fermion $\psi$ charged under both $\mathrm{U}(1)$ fields is introduced in the hidden sector which can be a subcomponent of the dark matter in the Universe. Stueckelberg mass terms between the two new gauge U(1) fields and the hypercharge gauge boson mediate the interactions between the standard model sector and the hidden sector. A remarkable collider signature of this model is the enhanced long-lived dark photon events at the LHC than the conventional dark photon models; the long-lived dark photons in the model can be discriminated from the background by measuring the time delay signal in the precision timing detectors which are proposed to be installed in the LHC upgrades and have an $\mathcal{O}(10)$ pico-second detection efficiency. Searches with current LHCb data are also investigated. Various experimental constraints on the model including collider constraints and cosmological constraints are also discussed.

Keywords: Beyond Standard Model, Gauge Symmetry

ARXIV EPRINT: 1912.00422 


\section{Contents}

1 Introduction 1

2 The model 2

3 Experimental constraints 3

$\begin{array}{lll}4 & \text { Timing detector } & 7\end{array}$

$\begin{array}{lll}5 & \mathrm{LHCb} & 12\end{array}$

$\begin{array}{lll}6 & \text { Summary } & 13\end{array}$

A Decay and lifetime of dark photon $A^{\prime} \quad 14$

$\begin{array}{ll}\text { B Approximated couplings } & 15\end{array}$

\section{Introduction}

Recently, particles with a long lifetime have been studied extensively at colliders (see e.g. ref. [1] for a review). At the large hadron collider (LHC), the long-lived particles have been searched for in channels with displaced dilepton vertices [2-6] and in channels with displaced jets vertices [7-15]. Searches for displaced vertices of collimated leptons or light hadrons with low $p_{T}$, which originate from light neutral particles such as dark photons, have been carried out at the LHC, e.g. by the ATLAS collaboration [16, 17].

Typically, for a particle to be considered as a long-lived particle at the LHC, the decay length has to be larger than $\mathcal{O}(1) \mathrm{mm}$ so that the displaced vertex can be detected by the spatial resolution of the LHC detectors. Thus, the coupling strength between longlived particles and the standard model (SM) particles is usually significantly reduced. For example, an electrophilic vector boson $A^{\prime}$ that couples with electron via $g A_{\mu}^{\prime} \bar{e} \gamma^{\mu} e$ has a decay width $\Gamma=g^{2} m_{A^{\prime}} /(12 \pi)$ and a decay length $d=\gamma v \tau$, where $v(\tau)$ is the velocity (lifetime) of the vector boson $A^{\prime}$, and $\gamma$ is the Lorentz boost factor. For such a particle to have a macroscopic decay length so that it can give rise to a long-lived particle signature at the LHC, the decay coupling is typically small. For example, consider a typical decay length as $d \simeq 1 \mathrm{~m}$, one has $g \sim \mathcal{O}\left(10^{-6}\right)$ for the case where $m_{A^{\prime}}=1 \mathrm{GeV}$ and $\gamma=100$. Thus simple long-lived vector boson models usually lead to a suppressed LHC cross section due to the small coupling constant needed for the large decay length.

In this paper, we construct a model that predicts a long-lived dark photon (LLDP) with a $\mathrm{GeV}$ scale mass. Unlike many other dark photon models, the production cross section of the GeV LLDP in our model at the LHC is not suppressed. This is because the production 
process of the LLDP is different from its decay process. We use the Stueckelberg mechanism to mediate the interaction between the hidden sector and the SM sector; the production and decay processes of the LLDPs are mediated by different Stueckelberg mass terms.

Recently, models in which LLDPs can have a sizable collider signal have been proposed in the literature. For example, ref. [18] introduced a second boson with couplings to both SM quarks and the hidden fermion to produce dark photons at colliders. Ref. [19] used a dimension-five operator between a scalar $\mathrm{SU}(2)_{L}$ triplet, the $\mathrm{SM} \mathrm{SU}(2)_{L}$ gauge bosons and the dark gauge boson to generate a non-abelian kinetic mixing term, which can enhance the LLDP signal. Potential large LLDP collider signals can also arise via top-partner decays [20], or via a Higgs portal interaction to the hidden QED [21].

\section{The model}

We introduce two Abelian gauge groups in the hidden sector: $\mathrm{U}(1)_{F}$ with gauge boson $X^{\mu}$ and $\mathrm{U}(1)_{W}$ with gauge boson $C^{\mu}$. We use the Stueckelberg mechanism to provide masses to the two new gauge bosons in the hidden sector, and also to mediate the interactions between the hidden sector and the SM sector [23-28]. The Lagrangian for the extension is given by $\mathcal{L}=\mathcal{L}_{F}+\mathcal{L}_{W}$ where

$$
\begin{aligned}
-4 \mathcal{L}_{F} & =X_{\mu \nu}^{2}+2\left(\partial_{\mu} \sigma_{1}+m_{1} \epsilon_{1} B_{\mu}+m_{1} X_{\mu}\right)^{2}, \\
-4 \mathcal{L}_{W} & =C_{\mu \nu}^{2}+2\left(\partial_{\mu} \sigma_{2}+m_{2} \epsilon_{2} B_{\mu}+m_{2} C_{\mu}\right)^{2} .
\end{aligned}
$$

Here $B_{\mu}$ is the hypercharge boson in the SM, $\sigma_{1}$ and $\sigma_{2}$ are the axion fields in the Stueckelberg mechanism, and $m_{1}, m_{2}, m_{1} \epsilon_{1}$, and $m_{2} \epsilon_{2}$ are mass terms in the Stueckelberg mechanism. ${ }^{1}$ The dimensionless parameters $\epsilon_{1}$ and $\epsilon_{2}$ are assumed to be small in our analysis: we set $\epsilon_{1} \sim \mathcal{O}\left(10^{-7}\right)$ so that an LLDP at the LHC can arise; we set $\epsilon_{2} \sim \mathcal{O}\left(10^{-2}\right)$ in order to obtain a significant LHC production cross section while satisfying various experimental constraints. $\mathcal{L}_{F}$ is invariant under $\mathrm{U}(1)_{Y}$ gauge transformations $\delta_{Y} B_{\mu}=\partial_{\mu} \lambda_{B}$ and $\delta_{Y} \sigma_{1}=-m_{1} \epsilon_{1} \lambda_{B} ; \mathcal{L}_{F}$ is also invariant under $\mathrm{U}(1)_{F}$ gauge transformation $\delta_{F} X_{\mu}=\partial_{\mu} \lambda_{X}$, and $\delta_{F} \sigma_{1}=-m_{1} \lambda_{X}$. Similarly, $\mathcal{L}_{W}$ is gauge invariant under both $\mathrm{U}(1)_{Y}$ and $\mathrm{U}(1)_{W}$.

In the hidden sector, we further introduce one Dirac fermion $\psi$ that is charged under both $\mathrm{U}(1)_{F}$ and $\mathrm{U}(1)_{W}$. Vector current interactions between the Dirac fermion and the gauge bosons in the hidden sector are assumed, i.e., $g_{F} \bar{\psi} \gamma^{\mu} \psi X_{\mu}+g_{W} \bar{\psi} \gamma^{\mu} \psi C_{\mu}$ where $g_{F}$ and $g_{W}$ are the gauge couplings for $\mathrm{U}(1)_{F}$ and $\mathrm{U}(1)_{W}$ respectively.

The two by two mass matrix in the neutral gauge boson sector in the SM is now extended to a four by four mass matrix, which, in the gauge basis $V=\left(C, X, B, A^{3}\right)$, is given by

$$
M^{2}=\left(\begin{array}{cccc}
m_{2}^{2} & 0 & m_{2}^{2} \epsilon_{2} & 0 \\
0 & m_{1}^{2} & m_{1}^{2} \epsilon_{1} & 0 \\
m_{2}^{2} \epsilon_{2} & m_{1}^{2} \epsilon_{1} & \sum_{i=1}^{2} m_{i}^{2} \epsilon_{i}^{2}+\frac{g^{\prime 2} v^{2}}{4} & -\frac{g^{\prime} g v^{2}}{4} \\
0 & 0 & -\frac{g^{\prime} g v^{2}}{4} & \frac{g^{2} v^{2}}{4}
\end{array}\right)
$$

\footnotetext{
${ }^{1}$ Two additional mass parameters can be present in the Lagrangian so that $-4 \mathcal{L}_{F}=X_{\mu \nu}^{2}+2\left(\partial_{\mu} \sigma_{1}+\right.$ $\left.m_{1} \epsilon_{1} B_{\mu}+m_{1} X_{\mu}+m_{1}^{\prime} C_{\mu}\right)^{2}$, and $-4 \mathcal{L}_{W}=C_{\mu \nu}^{2}+2\left(\partial_{\mu} \sigma_{2}+m_{2} \epsilon_{2} B_{\mu}+m_{2} C_{\mu}+m_{2}^{\prime} X_{\mu}\right)^{2}$. In our analysis we take both $m_{1}^{\prime}$ and $m_{2}^{\prime}$ to be negligible for simplicity.
} 
where $g$ and $g^{\prime}$ are gauge couplings for the SM SU(2) $L$ and $\mathrm{U}(1)_{Y}$ gauge groups respectively, $A^{3}$ is the third component of the $\mathrm{SU}(2)_{L}$ gauge bosons, and $v$ is the Higgs vacuum expectation value. The determinant of the mass square matrix vanishes which ensures the existence of a massless mode to be identified as the SM photon.

The mass matrix can be diagonalized via an orthogonal transformation $\mathcal{O}$ such that $\mathcal{O}^{T} M^{2} \mathcal{O}=\operatorname{diag}\left(m_{Z^{\prime}}^{2}, m_{A^{\prime}}^{2}, m_{Z}^{2}, 0\right)$; the mass basis $E=\left(Z^{\prime}, A^{\prime}, Z, A\right)$ is related to the gauge basis $V$ via $E_{i}=\mathcal{O}_{j i} V_{j}$. In the mass basis, $A$ is the SM photon, $Z$ is the SM $Z$ boson, $A^{\prime}$ is the dark photon with a $\mathrm{GeV}$-scale mass and $Z^{\prime}$ is the heavy boson with a $\mathrm{TeV}$-scale mass. Diagonalization of the mass matrix leads to interactions between $Z / A$ in the SM to the fermion $\psi$ in the hidden sector, and also interactions between $Z^{\prime} / A^{\prime}$ in the hidden sector and SM fermions $f$; both $\psi-Z / A$ and $f-Z^{\prime} / A^{\prime}$ couplings are suppressed by the small $\epsilon_{1}$ and $\epsilon_{2}$ parameters, and vanish in the $\epsilon_{1}=0=\epsilon_{2}$ limit.

We parameterize the interactions between the fermions and the mass eigenstates of the neutral bosons via

$$
\bar{f} \gamma_{\mu}\left(v_{i}^{f}-\gamma_{5} a_{i}^{f}\right) f E_{i}^{\mu}+v_{i}^{\psi} \bar{\psi} \gamma_{\mu} \psi E_{i}^{\mu}
$$

where the vector and axial-vector couplings are given by

$$
\begin{aligned}
v_{i}^{f} & =\left(g \mathcal{O}_{4 i}-g^{\prime} \mathcal{O}_{3 i}\right) T_{f}^{3} / 2+g^{\prime} \mathcal{O}_{3 i} Q_{f}, \\
a_{i}^{f} & =\left(g \mathcal{O}_{4 i}-g^{\prime} \mathcal{O}_{3 i}\right) T_{f}^{3} / 2, \\
v_{i}^{\psi} & =g_{W} \mathcal{O}_{1 i}+g_{F} \mathcal{O}_{2 i},
\end{aligned}
$$

with $Q_{f}$ is the electric charge of fermion $f, T_{f}^{3}$ is the quantum number of the left-hand chiral component of the fermion $f$ under $\mathrm{SU}(2)_{L}$.

The eigenvector corresponding to the photon, the massless eigen mode, is $\mathcal{O}_{j 4}=N\left(-\epsilon_{2},-\epsilon_{1}, 1, g^{\prime} / g\right)$ where $N$ is the normalization. The SM fermion couplings to the photon are thus given by $v_{4}^{f}=e Q_{f}$ and $a_{4}^{f}=0$ where the coupling constant $e=g^{\prime} N$. As in SM, the photon here couples to the SM fermions only via the vector coupling that is proportional to the electric charge of the fermion. The coupling constant $e$ is now related to the gauge couplings $g$ and $g^{\prime}$ via

$$
\frac{1}{e^{2}}=\frac{1}{g^{2}}+\frac{1+\epsilon_{1}^{2}+\epsilon_{2}^{2}}{g^{\prime 2}} \equiv \frac{1}{g^{2}}+\frac{1}{g_{\mathrm{SM}}^{\prime 2}}
$$

where $g_{\mathrm{SM}}^{\prime}$ is the effective coupling constant in the SM. The hidden fermion $\psi$ interacts with the SM photon via the vector coupling $v_{4}^{\psi}=-\left(e / g^{\prime}\right)\left(\epsilon_{2} g_{W}+\epsilon_{1} g_{F}\right) \equiv e \delta$ where $\delta$ is a very small electric charge which is often referred to as "millicharge" in the literature. We also present approximated expressions of the vector and axial-vector couplings for the three massive eigen modes in appendix B.

\section{Experimental constraints}

Here we discuss various constraints on the model, including electroweak constraints from LEP, constraints from LHC, and also cosmological constraints. 
In our analysis, we will fix most model parameters so that a sizable LHC signal is expected. We first discuss these default parameter values. The heavy $Z^{\prime}$ boson in our model mostly originates from the $\mathrm{U}(1)_{W}$ boson $C_{\mu}$; we choose $m_{2}=700 \mathrm{GeV}$ as the benchmark point in our analysis. ${ }^{2}$ In order to obtain a sufficient large $\psi \bar{\psi}$ production cross section at the LHC, we choose $g_{W}=1$; a relatively large $\mathrm{U}(1)_{F}$ coupling constant is also chosen, $g_{F}=1.5$, so that a rather sizable dark radiation rate for the $\psi$ particle can be achieved in the model. ${ }^{3}$ We use $c \tau=\hbar c / \Gamma=1 \mathrm{~m}$ as the characteristic value for the proper lifetime of the dark photon, where $\Gamma$ is the dark photon decay width; the dark photon decay widths are given in appendix A. We find that small modifications around $c \tau=1 \mathrm{~m}$ do not lead to significant changes in the collider signatures. The above values are the default ones used throughout the analysis, if not explicitly specified.

$Z$ invisible decay. The $Z$ invisible decay width is measured to be $\Gamma_{\mathrm{inv}}^{Z} \pm \delta \Gamma_{\mathrm{inv}}^{Z}=499 \mathrm{MeV}$ $\pm 1.5 \mathrm{MeV}[29]$. The $Z$ boson can decay into the $\psi \bar{\psi}$ final state, if $m_{Z}>2 m_{\psi}$, with a decay width

$$
\Gamma_{Z \rightarrow \psi \bar{\psi}}=\frac{m_{Z}}{12 \pi}\left(v_{3}^{\psi}\right)^{2} \sqrt{1-4 x_{\psi Z}}\left(1+2 x_{\psi Z}\right)
$$

where $x_{\psi Z} \equiv\left(m_{\psi} / m_{Z}\right)^{2}$, and $v_{3}^{\psi}$ is the vector coupling between the $Z$ boson and $\psi$, as given in eq. (2.5). Equating the invisible decay width due to the $\psi \bar{\psi}$ final state to the experimental uncertainty $\delta \Gamma_{\text {inv }}^{\mathrm{Z}}$, one obtains an upper bound on $v_{3}^{\psi}$, which is shown on figure 1. For light $\psi$ mass, one has $v_{3}^{\psi} \gtrsim 2.5 \times 10^{-2}$.

Electroweak constraint on the $Z$ mass. The mass of the $Z$ boson is modified due to the enlarged neutral gauge boson mass matrix, as given in eq. (2.1). For the parameter space of interest in our analysis, i.e., $\epsilon_{1} \ll \epsilon_{2}$, the mass shift on the $Z$ boson can be estimated as

$$
\left|\frac{\Delta m_{Z}}{m_{Z}}\right| \simeq \frac{\epsilon_{2}^{2}}{2} s_{W}^{2}\left(1-\frac{m_{Z}^{2}}{m_{2}^{2}}\right)^{-1},
$$

where $s_{W}^{2} \equiv \sin ^{2} \theta_{W}=0.22343$ [30], with $\theta_{W}$ being the weak rotation angle. We adopt the methodology in ref. [24] to estimate the electroweak constraints. The experimental uncertainty of the $Z$ mass is given by [24]

$$
\left[\frac{\delta m_{Z}}{m_{Z}}\right]^{2}=\left[\frac{c_{W}^{-2}-2 t_{W}^{2}}{\delta m_{W}^{-1} m_{W}}\right]^{2}+\frac{t_{W}^{4}(\delta \Delta r)^{2}}{4(1-\Delta r)^{2}}
$$

where $c_{W} \equiv \cos \theta_{W}$, and $t_{W} \equiv \tan \theta_{W}$. Here we take into account the recent analysis on the uncertainty of the $W$ boson mass, $m_{W} \pm \delta m_{W}=80.387 \pm 0.016 \mathrm{GeV}$ [30], and on the radiative correction, $\Delta r \pm \delta \Delta r=0.03672 \mp 0.00017 \pm 0.00008$ [30] where the first uncertainty is due to the top quark mass and the second is due to the fine structure constant $\alpha\left(m_{Z}\right)$ at the $m_{Z}$ scale. Adding in quadrature, we obtain $\delta \Delta r=0.00019$. Equating the mass shift

\footnotetext{
${ }^{2}$ The LLDP signal at the LHC is insensitive to the $m_{2}$ value when $m_{2}>\mathcal{O}(100) \mathrm{GeV}$.

${ }^{3}$ We set $g_{W}=1$ and $g_{F}=1.5$ at the $A^{\prime}$ mass scale. The RGE running of $g_{W}$ is insignificant, $\lesssim 5 \%$ increment on $g_{W}$ at the $Z^{\prime}$ mass scales.
} 


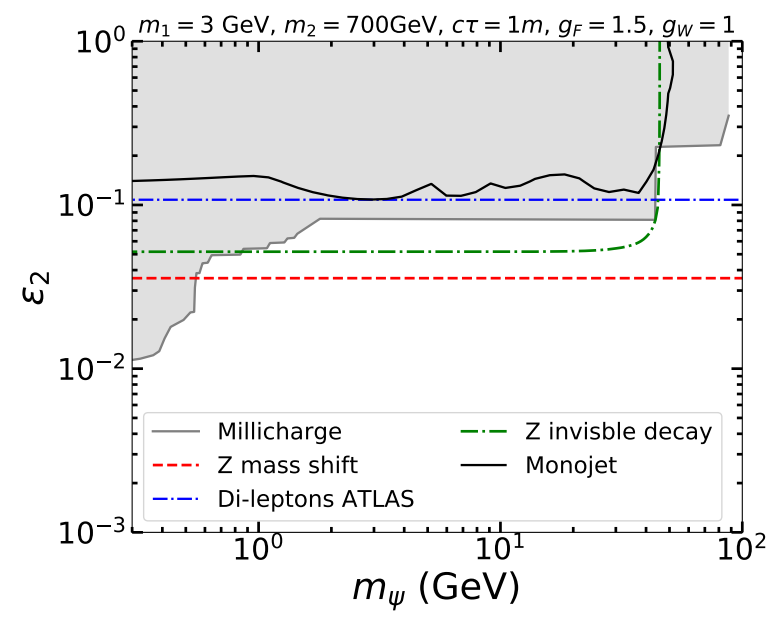

Figure 1. The $95 \%$ CL exclusion limits on $\epsilon_{2}$ as a function of $m_{\psi}$. We use the following parameters: $m_{1}=3 \mathrm{GeV}, m_{2}=700 \mathrm{GeV}, g_{F}=1.5, g_{W}=1.0$, and $c \tau=1 \mathrm{~m}$. Shown are the millicharged particle searches at the colliders (shaded light gray) [33], electroweak constraints due to the $Z$ mass shift (dashed red), the $Z$ invisible decay (dash-dotted green) [29], the di-lepton high mass resonance search at ATLAS (dash-dotted blue) [32], and the mono jet search at ATLAS (solid black) [34].

on the $Z$ boson given in eq. (3.2) to the experimental uncertainty, one obtains an upper bound on $\epsilon_{2}$

$$
\left|\epsilon_{2}\right| \lesssim 0.036 \sqrt{1-\left(m_{Z} / m_{2}\right)^{2}}
$$

The upper limit analyzed in this section using only the $Z$ mass constraint is similar to that from a global-fit analysis on a number of Z-pole observables [24]. Using the $Z$ mass constraint, we can also reproduce the limit obtained in a more recent electroweak global analysis [31]. Thus the method using only the $Z$ mass constraint provides a quick way to estimate the electroweak constraints.

Di-lepton constraint on $Z^{\prime}$ decays. Dilepton final states which are produced at the LHC from the heavy $Z^{\prime}$ boson via the Drell-Yan process, can be searched for by reconstructing their invariant mass. Figure 1 shows the $95 \%$ CL upper bound on $\epsilon_{2}$ in the dilepton channel from ATLAS [32].

Constraints on millicharge. In the parameter space of interest of the model, the hidden fermion $\psi$ possesses a millicharge $\delta \simeq \epsilon_{2} g_{W} / g^{\prime}$. The $\psi$ particle remains undetectable in typical particle detectors due to the minute electric charge. Figure 1 shows the collider constraints on millicharge in the $\left(m_{\psi}, \epsilon_{2}\right)$ plane [33]. The collider constraints on millicharge are the most stringent constraints on $\epsilon_{2}$ for $m_{\psi} \lesssim 0.6 \mathrm{GeV}$.

Because the $\psi$ particle is charged under $\mathrm{U}(1)_{W}$ and $\mathrm{U}(1)_{F}$ in the hidden sector, it is stable and thus can be a dark matter (DM) candidate. For the parameter space of interest, i.e., $m_{\psi}>m_{A^{\prime}}$, the annihilation channel into on-shell dark photons, $\psi \bar{\psi} \rightarrow A^{\prime} A^{\prime}$, is the dominant process for the relic abundance of the $\psi$ particle; the annihilation cross section 


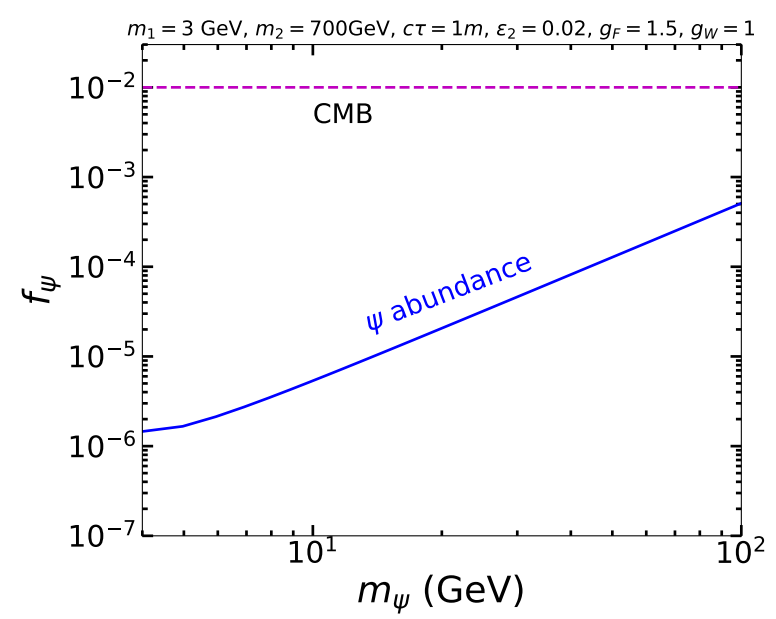

Figure 2. The fraction of $\psi$ to the total DM in the Universe as a function of the $\psi$ mass. Here, we take the canonical DM cross section is $\langle\sigma v\rangle_{\mathrm{DM}}=1 \mathrm{pb}$. The magenta dashed line represents the current limit from refs. [35-38].

can be approximated as follows [39]

$$
\langle\sigma v\rangle_{\psi \bar{\psi} \rightarrow A^{\prime} A^{\prime}} \simeq \frac{\left(v_{2}^{\psi}\right)^{4}}{16 \pi m_{\psi}^{2}} \frac{\left(1-r^{2}\right)^{3 / 2}}{\left(1-r^{2} / 2\right)^{2}}
$$

where $v_{2}^{\psi}$ is the coupling between the dark photon and $\psi$, as given in eq. (2.5), and $r=m_{A^{\prime}} / m_{\psi}$. We compute the ratio between the $\psi$ relic abundance and the total DM relic abundance via $f_{\psi}=2\langle\sigma v\rangle_{\mathrm{DM}} /\langle\sigma v\rangle_{\psi \bar{\psi} \rightarrow A^{\prime} A^{\prime}}$, where $\langle\sigma v\rangle_{\mathrm{DM}}=1 \mathrm{pb}$ is the canonical DM cross section, and the factor of 2 accounts for the Dirac nature of the $\psi$ particle. Because $v_{2}^{\psi} \simeq g_{F}$, the annihilation cross section given in eq. (3.5) is much larger than the canonical annihilation cross section needed for the cold DM relic density in the Universe [40], for the case $m_{A^{\prime}} \sim \mathcal{O}(1) \mathrm{GeV}$ and $m_{\psi} \sim \mathcal{O}(10) \mathrm{GeV}$. Thus, the contribution of the $\psi$ particle to the DM in the Universe is less $0.1 \%$ when $m_{\psi}<100 \mathrm{GeV}$, as shown in figure 2. This is consistent with the cosmological limits on millicharged DM, which constrain the fraction of the millicharged DM to be $\lesssim 1 \%$ of the total DM in the Universe [35-38]. The $\psi$ DM is efficiently stopped by the rock above underground labs of DM direct detection experiments, unless the millicharge is extremely small. Adapting the estimation in refs. [41, 42] for $1 \mathrm{~km}$ of rock, we found that the DM direct detection is only sensitive to the mixing parameter of $\epsilon_{2}<10^{-6}$ in our model. Thus the current underground DM direct detection experiments do not constrain the model.

Monojet constraints. Searches for invisible particles which are produced in association with an initial state radiation (ISR) jet have been carried out at ATLAS [34] and CMS [43]. Here we recast the ATLAS result [34] to set constraints on our model. We use MadGraph5 aMC@NLO (MG5) [44] to generate events for the process $p p \rightarrow \psi \bar{\psi} j$ which are then passed to Pythia 8 for showering and hadronization $[22,45,46]$. The Madanalysis 5 package $[47,48]$ is further used to analyze the ATLAS results [34]. We use the same detector cuts as in ref. [34]; the optimal selection region for our model is found to be in the window: 


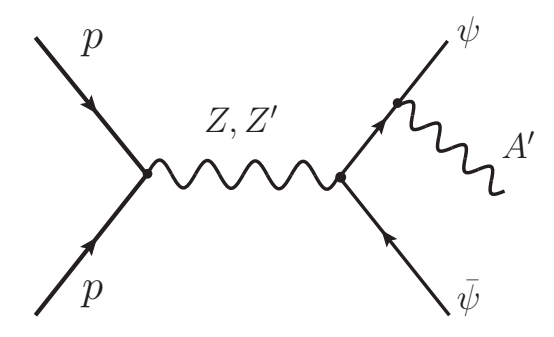

Figure 3. Feynman diagram for the dark photon production at the LHC.

$E_{T}^{\text {miss }} \in(300,350) \mathrm{GeV}$ (the EM2 region in ref. [34]). The 95\% CL exclusion limit on $\epsilon_{2}$ from the monojet channel in ATLAS [34] is shown in figure 1. When $m_{\psi}<m_{Z} / 2$, the $Z$ boson diagram, i.e., the $p p \rightarrow Z j \rightarrow \psi \bar{\psi} j$ process gives the dominant contribution to the monojet signal; when $m_{\psi}>m_{Z} / 2$, the $Z^{\prime}$ boson diagram, i.e., the $p p \rightarrow Z^{\prime} j \rightarrow \psi \bar{\psi} j$ becomes more important than the $Z$ process. Because of the large $Z^{\prime}$ mass, the $Z^{\prime}$ boson diagram is suppressed as compared to the $Z$ diagram. Thus the monojet channel only provides a comparable constraint to other constraints for $m_{\psi}<m_{Z} / 2$.

As shown in figure 1, the electroweak constraint on the $Z$ mass shift provides the best constraint to the parameter space of interest in our model, except for the $m_{\psi} \lesssim 0.6 \mathrm{GeV}$ region where collider constraints on millicharged particles become strong.

\section{Timing detector}

Recently, some precision timing detectors are proposed to be installed at CMS [49], ATLAS $[50,51]$ and LHCb [52]. These timing detectors, which aim to reduce the pile-up rate at the high luminosity LHC (HL-LHC), can also be used in long-lived particle searches [53-56]. In this analysis, we focus on one of the timing detectors, the minimum ionizing particle (MIP) timing detector to be installed to the CMS detector (hereafter MTD CMS) [49], which has a $\sim 30$ pico-second timing resolution. At the MTD CMS, the timing detection layer, which is proposed to be installed between the inner tracker and the electromagnetic calorimeter, is about $1.17 \mathrm{~m}$ away from the beam axis and $6.08 \mathrm{~m}$ long in the beam axis direction. A time delay at the LHC due to long-lived particles can be measured by the new precision timing detectors, which can enhance the collider sensitivity to such models [53].

At the LHC, the hidden sector particle $\psi$ can be pair-produced via $p p \rightarrow Z / Z^{\prime} \rightarrow \bar{\psi} \psi$, which subsequently radiates dark photons $\psi \rightarrow \psi A^{\prime}$; the corresponding Feynman diagram is shown in figure 3. Due to the feeble interaction strength to SM fermions, the $A^{\prime}$ boson travels a macroscopic distance away from its production point and then decays into a pair of SM particles which are detected by the timing layers. Here we use the di-lepton final states measured by the timing layers to detect the LLDP in our model. The time delay between the leptons from the LLDP and SM particles produced at the primary vertex is given by

$$
\Delta t=L_{A^{\prime}} / v_{A^{\prime}}+L_{\ell}-L_{\mathrm{SM}},
$$


where the $L$ 's are the distances traveled by various particles and $v=c$ for the SM particles [53]. The time delay is significant if the LLDP moves non-relativistically.

We select the leading leptons with transverse momentum $p_{T}^{\ell}>3 \mathrm{GeV}$ to suppress faked signals from hadrons produced with low $p_{T}$ [57] and to ensure that the lepton is moving relativistically and its trajectory is not significantly bent [53]. The point where the dark photon decays is required to have a radial distance away from the beam axis of $0.2 \mathrm{~m}<$ $L_{A^{\prime}}^{T}<1.17 \mathrm{~m}$ and a longitudinal distance along the beam axis of $\left|z_{A^{\prime}}\right|<3.04 \mathrm{~m}$. Following ref. [53], an ISR jet with $p_{T}^{j}>30 \mathrm{GeV}$ and $\left|\eta_{j}\right|<2.5$ is required to time stamp the hard collision. The time delay is required to be $\Delta t>1.2 \mathrm{~ns}$ in order to suppress the background.

The dominant SM backgrounds come from the multi trackless jets in the same-vertex (SV) hard collisions and in the pile-up (PU) events [53], as well as photons in SV hard collisions [54]. The SV background arises because of the finite timing resolution; the PU background is due to the fact that within one bunch crossing, two hard collisions occurring at two different times can lead to a time delay signal.

We compute the dijet events at the LHC with $\sqrt{s}=13 \mathrm{TeV}$ by using MG5 [44] and also Pythia 8 [45]. We select events in which the leading jet has $p_{T}>30 \mathrm{GeV}$ and $|\eta(j)|<2.5$ to time stamp the primary collision, and the subleading jets have $p_{T}^{j}>3 \mathrm{GeV}$ and $|\eta(j)|<2.5$. The inclusive jet cross section is $\sigma_{j} \approx 1 \times 10^{8} \mathrm{pb}$, under these detector cuts.

The inclusive photon production cross section at NLO is $\sigma_{\gamma} \approx 2 \times 10^{8} \mathrm{pb}$ at the LHC with $\sqrt{s}=13 \mathrm{TeV}$, by using JETPHOX [58] with the CT10 PDF. The detector cuts are $p_{T}^{\gamma}>3 \mathrm{GeV}$ and $\left|\eta^{\gamma}\right|<2.5$ for photon and $p_{T}^{j}>30 \mathrm{GeV}$ and $\left|\eta^{j}\right|<2.5$ for the leading jet.

At the $13 \mathrm{TeV}$ LHC, the SV background events can be estimated as [53, 54]

$$
N_{\mathrm{SV}}=\sigma_{\gamma} \mathcal{L}+\sigma_{j} \mathcal{L} f_{\gamma} \sim 6 \times 10^{14}
$$

where $\mathcal{L}=3 \mathrm{ab}^{-1}$ is the integrated luminosity, and $f_{\gamma} \approx 10^{-4}$ is the rate of a jet to fake a photon or a lepton [54]. The PU background events can be estimated as [53, 54]

$$
N_{\mathrm{PU}}=\sigma_{j} \mathcal{L}\left(n_{\mathrm{PU}} \frac{\sigma_{j}^{\prime}}{\sigma_{\mathrm{inc}}}\right) f_{\gamma} f_{j} \sim 3.75 \times 10^{9},
$$

where $f_{j} \sim 10^{-3}[54]$ is the rate for the jet to be trackless, $\sigma_{j}^{\prime} \approx 1 \times 10^{11} \mathrm{pb}$ is the dijet cross section with the requirement on all jets of $p_{T}^{j}>3 \mathrm{GeV}$ and $|\eta(j)|<2.5, \sigma_{\text {inc }}=80$ $\mathrm{mb}[59]$ is the inelastic cross section of $p p$ collisions at $13 \mathrm{TeV}$ and $n_{\mathrm{PU}} \approx 100[60]$ is the average pile-up number at the HL-LHC.

The time delay distribution of the SM background can be described by a Gaussian distribution [53]

$$
\frac{d \mathcal{P}(\Delta t)}{d \Delta t}=\frac{1}{\sqrt{2 \pi} \delta_{t}} e^{\frac{-\Delta t^{2}}{2 \delta_{t}^{2}}}
$$

where $\delta_{t}$ is the time spread. For the PU background, the time spread, $\delta_{t}=190 \mathrm{ps}$, is determined by the beam property; for the SV background, $\delta_{t}=30 \mathrm{ps}$, is determined by the time resolution [49]. We find that under the detector cut $\Delta t>1 \mathrm{~ns}$, the SV background is negligible and the PU background is about 260 with $\mathcal{L}=3 \mathrm{ab}^{-1}$; the PU background also becomes negligible, $N_{\mathrm{PU}} \lesssim 0.5$, if the time delay $\Delta t>1.2 \mathrm{~ns}$ is required. Thus, we take $\Delta t>1.2 \mathrm{~ns}$ as the detector cut in our analysis. 


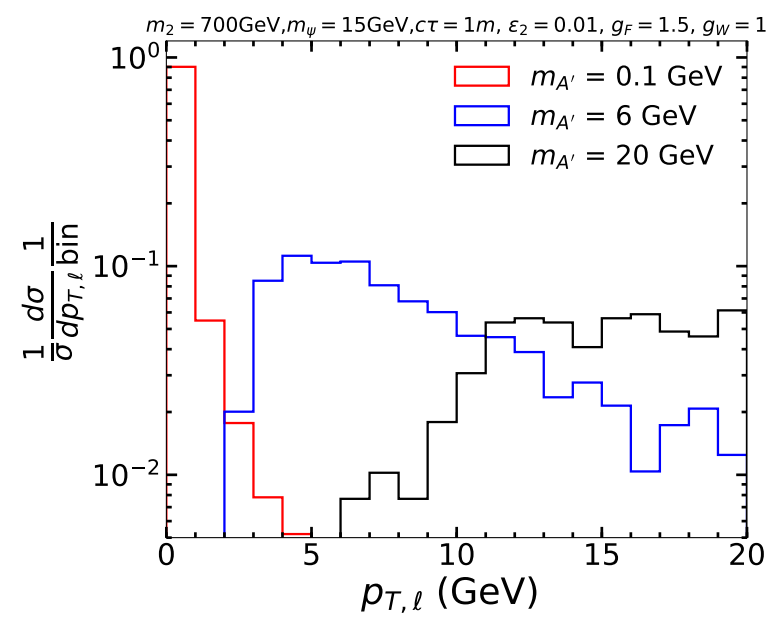

Figure 4. The $p_{T}$ distribution of the leading lepton. We choose $m_{2}=700 \mathrm{GeV}, m_{\psi}=15 \mathrm{GeV}$, $c \tau=1 \mathrm{~m}, \epsilon_{2}=0.01, g_{F}=1.5$ and $g_{W}=1.0$. The red, blue and black lines indicate the dark photon mass of $0.1 \mathrm{GeV}, 6 \mathrm{GeV}$ and $20 \mathrm{GeV}$ respectively.

We perform a full MC simulation and study the efficiency of the detector cuts in the parameter space of interest. We first implement the model into the FeynRules package [62] and pass the UFO model file into MG5 [44] to generate $8 \times 10^{4}$ events of the $\psi$ pair production associated with the time stamping ISR jet i.e $p p \rightarrow \psi \bar{\psi} j$. The dark showering is simulated in Pythia 8 [22, 45, 46].

Figure 4 shows the transverse momentum distribution of the leading lepton, for three different dark photon masses. We choose $m_{2}=700 \mathrm{GeV}, m_{\psi}=15 \mathrm{GeV}, c \tau=1 \mathrm{~m}$, $\epsilon_{2}=0.01, g_{F}=1.5$ and $g_{W}=1.0$, as the benchmark point. The final state leptons from dark photon decays are generally not very energetic in the models shown in figure 4 . In particular, the lepton events are highly suppressed under the detector cut $p_{T}>3 \mathrm{GeV}$, for the $0.1 \mathrm{GeV}$ dark photon case.

Figure 5 shows the distribution of the time delay $\Delta t$. The model parameters in figure 5 are the same as in figure 4. The SM backgrounds are negligible when the time delay $\Delta t>1.2 \mathrm{~ns}$. When the dark photon becomes heavier, more events with a larger time delay appear, as shown in figure 5 , since in this case, the dark photon has a higher probability to move non-relativistically. The increase of the events with the larger time delay, however, is offset by the smaller dark photon radiation rate of the heavier dark photon.

Figure 6 shows the cut efficiency as a function of $m_{A^{\prime}}$ and $m_{\psi}$, where 1370 grid points are simulated. We set $m_{2}=700 \mathrm{GeV}, c \tau=1 \mathrm{~m}, \epsilon_{2}=0.01, g_{F}=1.5$ and $g_{W}=1.0$. As shown in figure 4 , the detector cut: $p_{T}>3 \mathrm{GeV}$ for the leading lepton, significantly reduces the efficiency for light dark photon mass. The low efficiency in the heavy mass region in figure 6 is primarily due to the low radiation rate [63]. It turns out that the region with significant cut efficiency has $5 \mathrm{GeV}<m_{A^{\prime}}, m_{\psi}<35 \mathrm{GeV}$, with the highest efficiency $\sim 0.18 \%$. We note that, for the $m_{A^{\prime}}>2 m_{\psi}$ region, the dark photon is no longer a long-lived particle since it can decay into a pair of $\psi$. 


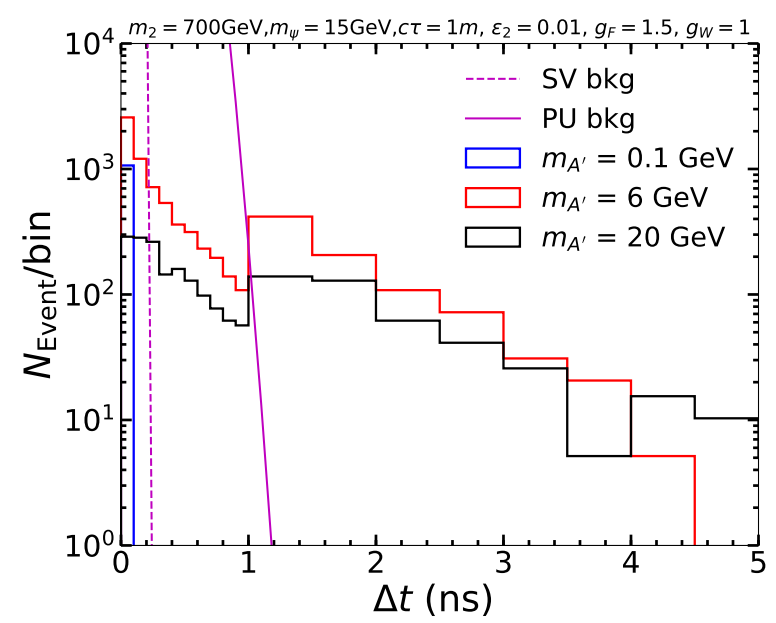

Figure 5. The distribution of the time delay $\Delta t$, with the integrated luminosity $\mathcal{L}=3 \mathrm{ab}^{-1}$. $p_{T}>3 \mathrm{GeV}$ is required for the leading lepton. The bin width is $0.5(0.1) \mathrm{ns}$ for $\Delta t>1 \mathrm{~ns}(\Delta t<1$ ns). We choose $m_{2}=700 \mathrm{GeV}, m_{\psi}=15 \mathrm{GeV}, c \tau=1 \mathrm{~m}, \epsilon_{2}=0.01, g_{F}=1.5$ and $g_{W}=1.0$. The solid blue, red and black lines indicate the dark photon mass of $0.1 \mathrm{GeV}, 6 \mathrm{GeV}$ and $20 \mathrm{GeV}$. The solid and dashed magenta curves represent the PU and SV backgrounds respectively.

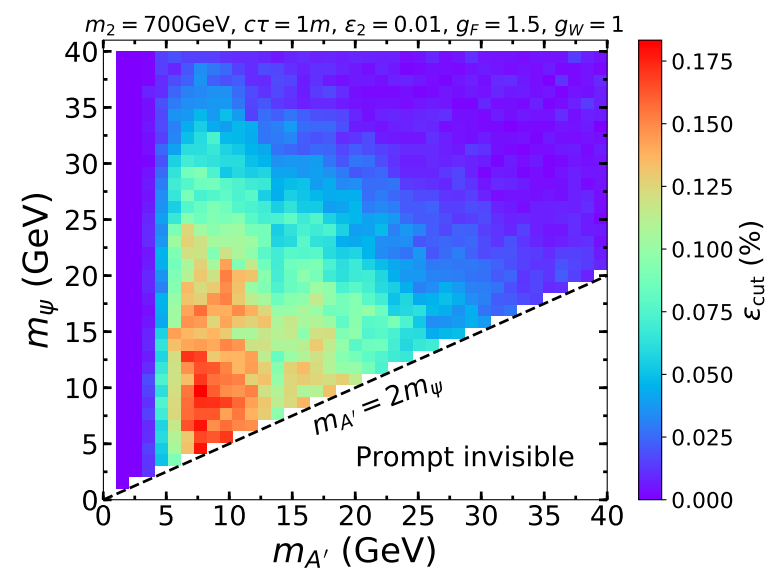

Figure 6. The cut efficiency $\epsilon_{\text {cut }}$ as a function of $m_{A^{\prime}}$ and $m_{\psi}$. We set $m_{2}=700 \mathrm{GeV}, c \tau=1 \mathrm{~m}$, $\epsilon_{2}=0.01, g_{F}=1.5$ and $g_{W}=1.0$.

Figure 7 shows the regions that can be probed at the $13 \mathrm{TeV}$ LHC, with the discovery criterion: $S=10$, as a function of the dark photon mass and the $\psi$ mass. The number of signal events is computed via $S=\epsilon_{\text {cut }} \mathcal{L} \sigma(p p \rightarrow \psi \bar{\psi} j)$, where $\mathcal{L}$ is the integrated luminosity, $\sigma(p p \rightarrow \psi \bar{\psi} j)$ is the production cross section at the LHC, and $\epsilon_{\text {cut }}$ is the cut efficiency as shown in figure 6 . The model parameters are the same as in figure 6 . The blue and magenta contours indicate the needed integrated luminosity to generate 10 signal events. Therefore, with an integrated luminosity of $70 \mathrm{fb}^{-1}$ at the HL-LHC, the LLDP can be discovered in the time delay channel in the mass region: $5 \mathrm{GeV}<m_{A^{\prime}}, m_{\psi}<21 \mathrm{GeV}$, with the rest of the model parameters fixed as in figure 6. A larger mass region: $3 \mathrm{GeV}<m_{A^{\prime}}, m_{\psi}<30 \mathrm{GeV}$, can be discovered if $250 \mathrm{fb}^{-1}$ data are accumulated at the HL-LHC. 


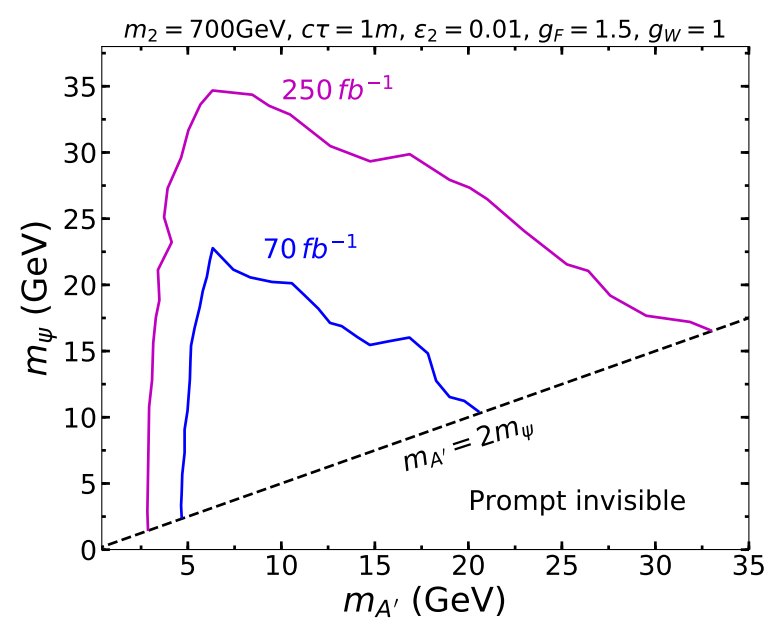

Figure 7. The contour of the expected signal events at the $13 \mathrm{TeV}$ LHC, as a function of $m_{A^{\prime}}$ and $m_{\psi}$. We choose $m_{2}=700 \mathrm{GeV}, c \tau=1 \mathrm{~m}, \epsilon_{2}=0.01, g_{F}=1.5$ and $g_{W}=1.0$. The blue and magenta contours indicate the needed integrated luminosity of $70 \mathrm{fb}^{-1}$ and $250 \mathrm{fb}^{-1}$ respectively, to generate 10 events.

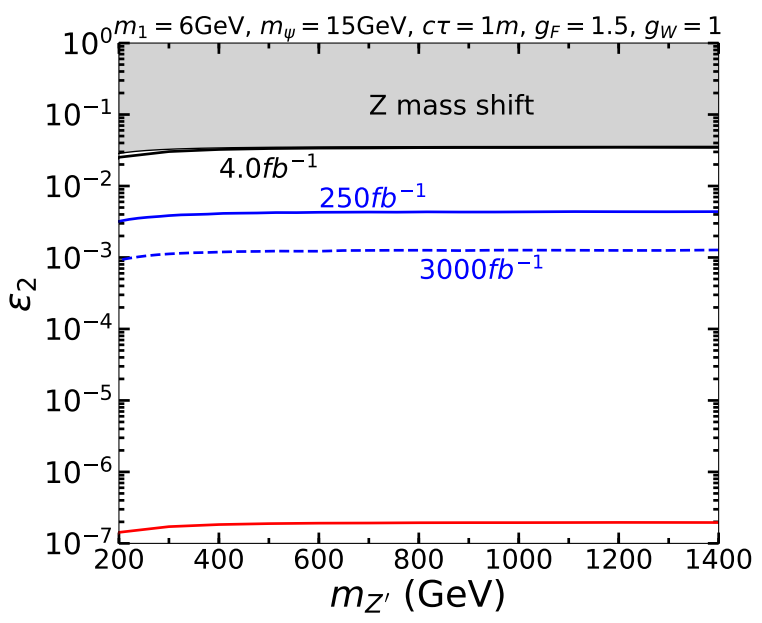

Figure 8. The integrated luminosity needed at the HL-LHC to probe the parameter region spanned by $m_{Z^{\prime}}$ and $\epsilon_{2}$. We choose $m_{1}=6 \mathrm{GeV}, m_{\psi}=15 \mathrm{GeV}, c \tau=1 \mathrm{~m}, g_{F}=1.5$ and $g_{W}=1$. The integrated luminosity needed are $\sim 4.0 \mathrm{fb}^{-1}$ (black solid), $250 \mathrm{fb}^{-1}$ (blue solid), and $3000 \mathrm{fb}^{-1}$ (blue dashed). The gray shaded region is excluded by the $Z$ mass shift constraint, as given in eq. (3.4). Below the red line, the dark photon production cross section via $\epsilon_{1}$ dominates.

Figure 8 shows the integrated luminosity needed to probe the parameter space spanned by $\epsilon_{2}$ and $m_{Z^{\prime}}$. We choose $m_{1}=6 \mathrm{GeV}, m_{\psi}=15 \mathrm{GeV}, c \tau=1 \mathrm{~m}, g_{F}=1.5$ and $g_{W}=1$ as a benchmark. With an integrated luminosity of $\sim 4.0 \mathrm{fb}^{-1}$, one can probe the $\epsilon_{2}$ value that saturates the electroweak constraint on the $Z$ mass shift. To discover a long-lived dark photon model in which $\epsilon_{2} \simeq 10^{-3}$, however, one needs about $3000 \mathrm{fb}^{-1}$ data at the HL-LHC. When $\epsilon_{2} \simeq \mathcal{O}\left(10^{-7}\right)$, the LLDP signal approaches the value in the conventional LLDP scenario, so that it is no longer enhanced by the production channel mediated by the $\epsilon_{2}$ parameter. 


\section{$5 \mathrm{LHCb}$}

Due to the excellent mass resolution $(7-20 \mathrm{MeV})$ and vertex resolution $(\sim 10 \mu \mathrm{m}$ on the transverse plane) as well as the ability for particle identification ( $\sim 90 \%$ for muons) $[65,66]$, the $\mathrm{LHCb}$ detector is able to discover new elusive particles beyond the SM. An upcoming upgrade with an increased luminosity and a more advanced trigger system with only software triggers will further improve the capability of the LHCb detector to probe new physics phenomena, such as LLDPs [66]. Recently, a search for LLDPs in the kinetic mixing model has been carried out at the LHCb via displaced muon pairs [64, 67, 68]. In our model, because LLDPs has a larger production cross section at the LHC than the conventional LLDP models, the LHCb search [64] can probe a much larger parameter space.

To analyze the LHCb constraints, we choose a benchmark point in which $m_{\psi}=5 \mathrm{GeV}$ and $\epsilon_{2}=0.01$, while the rest of the parameters take the default values. In this benchmark point, the $\psi$ production cross section, $\sigma(p p \rightarrow \psi \bar{\psi}) \sim 4.3 \mathrm{pb}$ which is dominated by the $Z$-boson exchange channel. We use MG5 [44] to generate the LHC events for each model point on the $\epsilon_{1}-m_{A^{\prime}}$ plane, which are then passed to Pythia $8[22,45,46]$ for showering (including showering in hidden sector) and hadronization.

We follow the LLDP search criteria in refs. $[64,68]$ to analyze the signal. In particular, we require the transverse distance of the dark photon decay vertex of $6 \mathrm{~mm}<l_{T}\left(A^{\prime}\right)<22 \mathrm{~mm}$ and the pseudo-rapidity of dark photons and muons of $2<\eta\left(A^{\prime}, \mu^{ \pm}\right)<4.5$. These requirements ensure that the displaced vertex is sufficiently separated from the beam line and registered in the Vertex Locator (VELO) where the dimuon can be reconstructed with good efficiency. Furthermore, in order to suppress the background from fake muons, we also require the momentum and transverse momentum of muons are greater than $10 \mathrm{GeV}$ and $0.5 \mathrm{GeV}$ respectively.

The dominant background includes the photon-conversion in the VELO, muons produced from b-hadron decay chains, and pions from $K_{s}^{0}$ decays which are misidentified as muons. Ref. [67] estimated the background events as $B=25$ for $\mathcal{L}=15 \mathrm{fb}^{-1}$, which is adopted in our analysis and also rescaled for the $\mathcal{L}=5.5 \mathrm{fb}^{-1}$ case. We compute the exclusion region by demanding that $S / \sqrt{B}>2.71$ where $S$ is the signal event number.

Figure 9 shows the LHCb exclusion region in the parameter space spanned by $\epsilon_{1}$ and the dark photon mass $m_{A^{\prime}}$. With the current luminosity $5.5 \mathrm{fb}^{-1}$, LHCb can probe the parameter space of our model: $200 \mathrm{MeV}<m_{A^{\prime}}<9 \mathrm{GeV}$ and $2 \times 10^{-7}<\epsilon_{1}<6 \times 10^{-5}$. The exclusion region in the conventional dark photon scenario is, however, much smaller, which is shown as two small gray islands at $\epsilon_{1} \sim\left(10^{-4}-10^{-5}\right)$. Thus, in our model, a significantly larger region of parameter space than the conventional dark photon model can be probed by the current LLDP search at the LHCb. A projected limit from the Run 3 data is also computed; LHCb can probe the parameter space: $200 \mathrm{MeV}<m_{A^{\prime}}<10 \mathrm{GeV}$ and $10^{-7}<\epsilon_{1}<10^{-4}$, if $15 \mathrm{fb}^{-1}$ integrated luminosity can be accumulated in the LHC Run 3 data. We note in passing that the shape of the exclusion contours is primarily due to the detector cut on the dark photon decay length: a smaller $\epsilon_{1}$ value is needed in the larger dark photon mass region so that the dark photon has the desired decay width to disintegrate in the VELO region. Also the dip at $m_{A^{\prime}} \simeq 0.8 \mathrm{GeV}$ is due to the $\omega$ resonance 


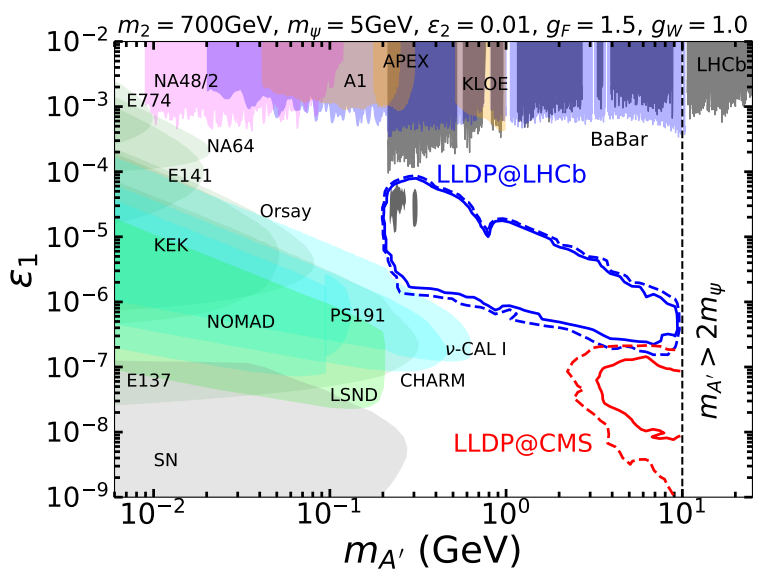

Figure 9. LHC current and future sensitivity contours to the LLDP parameter space spanned by $\epsilon_{1}$ and the dark photon mass. We take $m_{\psi}=5 \mathrm{GeV}$ and $\epsilon_{2}=0.01$. The blue solid and dashed contours indicate the regions probed by the LHCb with $5.5 \mathrm{fb}^{-1}$ and $15 \mathrm{fb}^{-1}$ data respectively. The regions probed by the future MTD CMS with $250 \mathrm{fb}^{-1}$ and $3000 \mathrm{fb}^{-1}$ data are shown as red solid and dashed contours respectively. The gray islands at $\epsilon_{1} \sim\left(10^{-4}-10^{-5}\right)$ are the LHCb exclusion regions for the conventional dark photon scenario [64]. Various experimental constraints on the conventional dark photon scenario are shown as color shaded regions.

which suppresses the $\operatorname{BR}\left(A^{\prime} \rightarrow \mu^{+} \mu^{-}\right)$. Figure 9 also shows the exclusion limits on the conventional dark photon from various experiments; the limits are taken from the Darkcast package [69].

Figure 9 also shows the sensitivities from the future MTD CMS detector via the time delay measurement. As mentioned before, the time delay signal from the final state leptons becomes more significant if the LLDPs have long lifetime and move non-relativistically. Therefore, the timing detector probes the heavy dark photon mass region with a smaller mixing parameter $\epsilon_{1}$ which are currently almost inaccessible at the LHCb. In particular, with the luminosity of $250 \mathrm{fb}^{-1}$ at the HL-LHC, the MTD CMS detector can probe the parameter space: $m_{A^{\prime}}>3.3 \mathrm{GeV}$ and $10^{-8}<\epsilon_{1}<10^{-7}$. An even larger parameter space in our model: $m_{A^{\prime}}>2.0 \mathrm{GeV}$ and $10^{-9}<\epsilon_{1}<2 \times 10^{-7}$, can be reached with $3000 \mathrm{fb}^{-1}$ data accumulated at the HL-LHC. Interestingly, this MTD CMS sensitivity region partly overlaps with the $\mathrm{LHCb}$ sensitivity region with $15 \mathrm{fb}^{-1}$ data. Thus, if the LLDP is discovered in this overlapped region, the timing detector can be used to verify the $\mathrm{LHCb}$ results. We note that, in the region of $m_{A^{\prime}}>2 m_{\psi}$, the dark photon will dominantly decay into $\psi$ so that it can no longer be searched for in the visible channel by the LHCb detector and the future precision timing detectors.

\section{Summary}

We construct a long-lived dark photon model which has an enhanced dark photon collider signal. We extend the standard model by a hidden sector which has two gauge bosons and one Dirac fermion $\psi$; the two gauge bosons interact with the SM sector via different Stueckelberg mass terms. The GeV-scale dark photon $A^{\prime}$ interacts with the SM fermions 
via a very small Stueckelberg mass term (parametrized by the dimensionless quantity $\epsilon_{1}$ ) such that it has a macroscopic decay length which can lead to a displaced vertex or a time delay signal at the LHC. The $\mathrm{TeV}$-scale $Z^{\prime}$ boson interacts with the SM via a relatively larger mass term (parametrized by the dimensionless quantity $\epsilon_{2}$ ). Because the dark photon $A^{\prime}$ is mainly produced at the $\mathrm{LHC}$ via the $\psi$ dark radiation processes in which the effective coupling strength is of the size of $\epsilon_{2}$ the LHC signal of $A^{\prime}$ is thus enhanced significantly.

Various experimental constraints on the model are analyzed, including the electroweak constraint on the $Z$ boson mass shift, the constraint from the $Z$ invisible decay, LHC constraints, collider constraints on millicharge, and cosmological constraints on millicharge. The electroweak constraint on the $Z$ mass turns out to be the most stringent one, which leads to an upper bound $\epsilon_{2} \lesssim 0.036$, in the parameter space of interest.

Two types of LHC signals from the LLDP in our model are investigated: the time delay signal measured by the precision timing detectors at the HL-LHC, and the current LHCb searches on LLDPs. If the LLDP is produced non-relativistically at the LHC, it has a significant time delay $\Delta t$, which can be measured by the precision timing detectors. Under the detector cut $\Delta t>1.2 \mathrm{~ns}$, the $\mathrm{SV}$ and PU backgrounds are found to be negligible. The parameter space of $3 \mathrm{GeV}<m_{A^{\prime}}, m_{\psi}<30 \mathrm{GeV}$ in our model is found to be probed by the timing detector with $250 \mathrm{fb}^{-1}$ data at the HL-LHC.

Due to the different search strategy, the current LHCb analysis is more sensitive to the lighter dark photon mass than the time delay searches. We found that the parameter space probed by the current $\mathrm{LHCb}$ analysis is much larger in our model than the conventional dark photon model investigated in the LHCb experimental analysis. A comparison between the LHCb search and the time delay search is also made; they typically probe different regions of the parameter space but can overlap in some small regions.

We note that a similar model as ours can be constructed by introducing two kinetic mixing parameters, of magnitude $\mathcal{O}\left(10^{-2}\right)$ and $\mathcal{O}\left(10^{-7}\right)$, which are responsible for dark photon production and decay processes, respectively.

\section{Acknowledgments}

We thank Jinhan Liang, Lei Zhang, and T.C. Yuan for helpful discussions and correspondence. The work is supported in part by the National Natural Science Foundation of China under Grant Nos. 11775109 and U1738134.

\section{A Decay and lifetime of dark photon $A^{\prime}$}

The dark photon leptonic decay width is given by

$$
\Gamma\left(A^{\prime} \rightarrow l^{+} l^{-}\right)=\frac{m_{A^{\prime}}}{12 \pi} \sqrt{1-4 \frac{m_{l}^{2}}{m_{A^{\prime}}^{2}}}\left[\left(1-4 \frac{m_{l}^{2}}{m_{A^{\prime}}^{2}}\right)\left|a_{2}^{l}\right|^{2}+\left(1+2 \frac{m_{l}^{2}}{m_{A^{\prime}}^{2}}\right)\left|v_{2}^{l}\right|^{2}\right],
$$

where $v_{2}^{l}$ and $a_{2}^{l}$ are the vector and axial-vector couplings between the dark photon and the leptons which are given in eqs. (2.3), (2.4). The hadronic decay width can be computed by

$$
\Gamma\left(A^{\prime} \rightarrow \text { hadrons }\right)=\Gamma\left(A^{\prime} \rightarrow \mu^{+} \mu^{-}\right) R\left(m_{A^{\prime}}^{2}\right),
$$



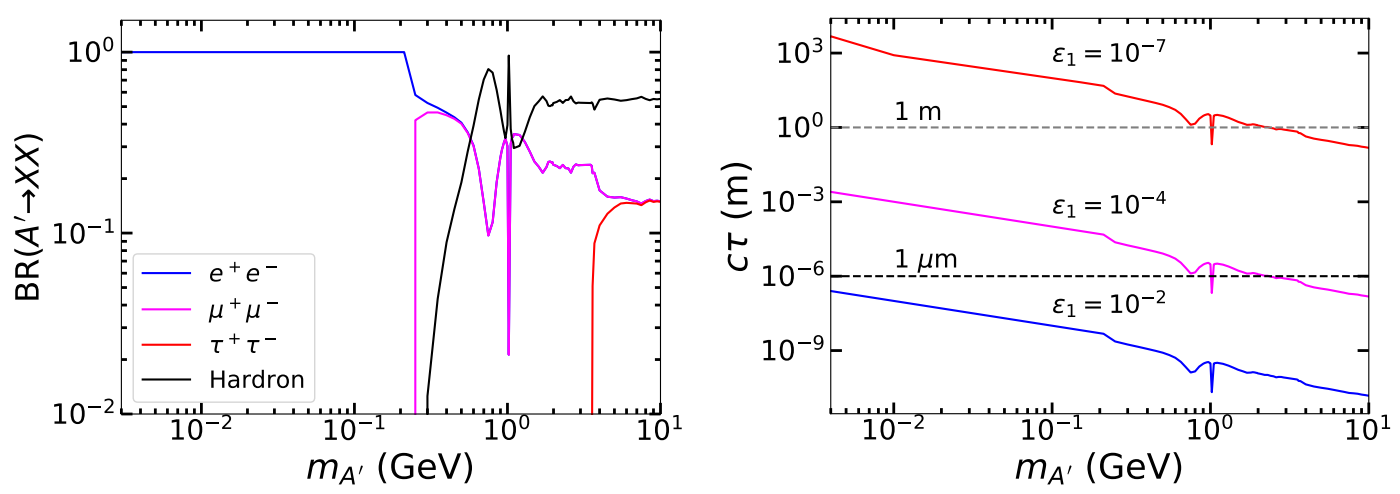

Figure 10. Left panel: branching ratio of the dark photon. Right panel: proper lifetime of the dark photon with various values of $\epsilon_{1}$. The dashed black line on the right panel figure indicates the criterion for the LHC prompt decay: $c \tau \leq 1 \mu \mathrm{m}$.

where $R\left(m_{A^{\prime}}^{2}\right) \equiv \sigma\left(e^{+} e^{-} \rightarrow\right.$ hadrons $) / \sigma\left(e^{+} e^{-} \rightarrow \mu^{+} \mu^{-}\right)$takes into account the effects of the dark photon mixing with the QCD vector mesons and is taken from ref. [30]. The $\Gamma\left(A^{\prime} \rightarrow \psi \bar{\psi}\right)$ can be computed by replacing the couplings and mass for leptons in eq. (A.1) with the ones for $\psi$. We note that below a few hundred $\mathrm{MeV}$, dark photon decays into the $e^{+} e^{-}$and $\mu^{+} \mu^{-}$pairs. In our analysis, since we consider $m_{A^{\prime}}<2 m_{\psi}$, the $\Gamma\left(A^{\prime} \rightarrow \psi \bar{\psi}\right)$ is kinematically forbidden.

Figure 10 shows the SM branching ratios of the dark photon decay and its proper lifetime with different $\epsilon_{1}$ values. We note that a particle is considered as a long-lived particle in particle colliders if its decay length is larger than the detector spatial resolution which can vary from $\mathcal{O}(10) \mu \mathrm{m}$ to $\mathcal{O}(10) \mathrm{mm}$ depending on the detectors.

\section{B Approximated couplings}

Here we provide approximated expressions of the vector and axial-vector couplings for the three massive gauge bosons, where $m_{1}=1 \mathrm{GeV}, m_{2}=700 \mathrm{GeV}, \epsilon_{1} \ll 1$ and $\epsilon_{2} \ll 1$. The approximated expressions of the vector and axial-vector couplings for the $Z^{\prime}$ boson are

$$
\begin{aligned}
v_{1}^{f} & \simeq\left(-0.18 T_{f}^{3}+0.35 Q_{f}\right) \epsilon_{2}, \\
a_{1}^{f} & \simeq-0.18 T_{f}^{3} \epsilon_{2}, \\
v_{1}^{\psi} & \simeq g_{W}+2.0 \times 10^{-6} \epsilon_{1} \epsilon_{2} g_{F} .
\end{aligned}
$$

The approximated expressions of the $A^{\prime}$ boson couplings are

$$
\begin{aligned}
v_{2}^{f} & \simeq\left(2.1 \times 10^{-5} T_{f}^{3}+0.27 Q_{f}\right) \epsilon_{1}, \\
a_{2}^{f} & \simeq 2.1 \times 10^{-5} T_{f}^{3} \epsilon_{1}, \\
v_{2}^{\psi} & \simeq g_{F}-0.78 \epsilon_{1} \epsilon_{2} g_{W} .
\end{aligned}
$$

The $A^{\prime}$ boson has a much larger vector coupling to the SM fermions than the axial-vector coupling, and the vector coupling is proportional to the electric charge when neglecting the 
small contribution from the isospin. The $A^{\prime}$ boson is called "dark photon" due to the fact it has a photon-like interaction to the SM fermions.

The approximated expressions of the $Z$ boson couplings are

$$
\begin{aligned}
v_{3}^{f} & \simeq v_{Z}^{f 0}+\left(0.041 \epsilon_{1}^{2}-1.4 \times 10^{-3} \epsilon_{2}^{2}\right) T_{f}^{3}-\left(0.18 \epsilon_{1}^{2}+0.034 \epsilon_{2}^{2}\right) Q_{f}, \\
a_{3}^{f} & \simeq a_{Z}^{f 0}+\left(0.041 \epsilon_{1}^{2}-1.4 \times 10^{-3} \epsilon_{2}^{2}\right) T_{f}^{3} \\
v_{3}^{\psi} & \simeq 0.48 \epsilon_{2} g_{W}-5.8 \times 10^{-5} \epsilon_{1} g_{F},
\end{aligned}
$$

where $v_{Z}^{f 0}$ and $a_{Z}^{f 0}$ are the SM values,

$$
\begin{aligned}
v_{Z}^{f 0} & =\frac{e}{2 s_{W} c_{W}}\left(T_{f}^{3}-2 s_{W}^{2} Q_{f}\right), \\
a_{Z}^{f 0} & =\frac{e}{2 s_{W} c_{W}} T_{f}^{3},
\end{aligned}
$$

where the weak rotation angle is given by $\tan \theta_{W}=g_{\mathrm{SM}}^{\prime} / g$ and $e=g s_{W}=g_{\mathrm{SM}}^{\prime} c_{W}$.

Open Access. This article is distributed under the terms of the Creative Commons Attribution License (CC-BY 4.0), which permits any use, distribution and reproduction in any medium, provided the original author(s) and source are credited.

\section{References}

[1] J. Alimena et al., Searching for Long-Lived Particles beyond the Standard Model at the Large Hadron Collider, arXiv:1903.04497 [INSPIRE].

[2] ATLAS collaboration, Search for massive, long-lived particles using multitrack displaced vertices or displaced lepton pairs in pp collisions at $\sqrt{s}=8 \mathrm{TeV}$ with the ATLAS detector, Phys. Rev. D 92 (2015) 072004 [arXiv: 1504.05162] [INSPIRE].

[3] ATLAS collaboration, Search for long-lived particles in final states with displaced dimuon vertices in pp collisions at $\sqrt{s}=13 \mathrm{TeV}$ with the ATLAS detector, Phys. Rev. D 99 (2019) 012001 [arXiv: 1808.03057] [INSPIRE].

[4] CMS collaboration, Search for long-lived particles that decay into final states containing two electrons or two muons in proton-proton collisions at $\sqrt{s}=8 \mathrm{TeV}$, Phys. Rev. D 91 (2015) 052012 [arXiv: 1411.6977] [INSPIRE].

[5] CMS collaboration, Search for Displaced Supersymmetry in events with an electron and a muon with large impact parameters, Phys. Rev. Lett. 114 (2015) 061801 [arXiv:1409.4789] [INSPIRE].

[6] ATLAS collaboration, Search for displaced vertices of oppositely charged leptons from decays of long-lived particles in pp collisions at $\sqrt{s}=13 \mathrm{TeV}$ with the ATLAS detector, Phys. Lett. B 801 (2020) 135114 [arXiv: 1907.10037] [INSPIRE].

[7] ATLAS collaboration, Search for decays of stopped, long-lived particles from $7 \mathrm{TeV} p p$ collisions with the ATLAS detector, Eur. Phys. J. C 72 (2012) 1965 [arXiv:1201.5595] [INSPIRE].

[8] CMS collaboration, Search for heavy long-lived charged particles in pp collisions at $\sqrt{s}=7$ TeV, Phys. Lett. B 713 (2012) 408 [arXiv:1205.0272] [INSPIRE]. 
[9] ATLAS collaboration, Search for long-lived stopped R-hadrons decaying out-of-time with pp collisions using the ATLAS detector, Phys. Rev. D 88 (2013) 112003 [arXiv:1310.6584] [INSPIRE].

[10] CMS collaboration, Search for Decays of Stopped Long-Lived Particles Produced in Proton-Proton Collisions at $\sqrt{s}=8$ TeV, Eur. Phys. J. C 75 (2015) 151 [arXiv: 1501.05603] [INSPIRE].

[11] ATLAS collaboration, Search for metastable heavy charged particles with large ionization energy loss in pp collisions at $\sqrt{s}=13$ TeV using the ATLAS experiment, Phys. Rev. D 93 (2016) 112015 [arXiv: 1604.04520] [INSPIRE].

[12] CMS collaboration, Search for long-lived charged particles in proton-proton collisions at $\sqrt{s}=13$ TeV, Phys. Rev. D 94 (2016) 112004 [arXiv:1609.08382] [InSPIRE].

[13] ATLAS collaboration, Search for long-lived, massive particles in events with displaced vertices and missing transverse momentum in $\sqrt{s}=13 \mathrm{TeV} p p$ collisions with the ATLAS detector, Phys. Rev. D 97 (2018) 052012 [arXiv:1710.04901] [INSPIRE].

[14] ATLAS collaboration, Search for long-lived neutral particles in pp collisions at $\sqrt{s}=13 \mathrm{TeV}$ that decay into displaced hadronic jets in the ATLAS calorimeter, Eur. Phys. J. C 79 (2019) 481 [arXiv: 1902.03094 ] [INSPIRE].

[15] CMS collaboration, Search for long-lived particles using delayed jets and missing transverse momentum with proton-proton collisions at $\sqrt{s}=13 \mathrm{TeV}$, CMS-PAS-EXO-19-001.

[16] ATLAS collaboration, Search for long-lived neutral particles decaying into lepton jets in proton-proton collisions at $\sqrt{s}=8 \mathrm{TeV}$ with the ATLAS detector, JHEP 11 (2014) 088 [arXiv: 1409.0746] [INSPIRE].

[17] ATLAS collaboration, Search for light long-lived neutral particles produced in pp collisions at $\sqrt{s}=13 \mathrm{TeV}$ and decaying into collimated leptons or light hadrons with the ATLAS detector, arXiv: 1909.01246 [INSPIRE].

[18] M. Buschmann, J. Kopp, J. Liu and P.A.N. Machado, Lepton Jets from Radiating Dark Matter, JHEP 07 (2015) 045 [arXiv:1505.07459] [INSPIRE].

[19] C.A. Argüelles, X.-G. He, G. Ovanesyan, T. Peng and M.J. Ramsey-Musolf, Dark Gauge Bosons: LHC Signatures of Non-Abelian Kinetic Mixing, Phys. Lett. B 770 (2017) 101 [arXiv: 1604.00044] [INSPIRE].

[20] J.H. Kim, S.D. Lane, H.-S. Lee, I.M. Lewis and M. Sullivan, Searching for Dark Photons with Maverick Top Partners, Phys. Rev. D 101 (2020) 035041 [arXiv: 1904.05893] [INSPIRE].

[21] A. Krovi, I. Low and Y. Zhang, Higgs Portal to Dark QED, arXiv:1909.07987 [InSPIRE].

[22] L. Carloni and T. Sjöstrand, Visible Effects of Invisible Hidden Valley Radiation, JHEP 09 (2010) 105 [arXiv: 1006.2911] [INSPIRE].

[23] B. Körs and P. Nath, Aspects of the Stueckelberg extension, JHEP 07 (2005) 069 [hep-ph/0503208] [INSPIRE].

[24] D. Feldman, Z. Liu and P. Nath, Probing a very narrow Z' boson with CDF and D0 data, Phys. Rev. Lett. 97 (2006) 021801 [hep-ph/0603039] [INSPIRE].

[25] D. Feldman, Z. Liu and P. Nath, The Stueckelberg $Z^{\prime}$ at the LHC: Discovery Potential, Signature Spaces and Model Discrimination, JHEP 11 (2006) 007 [hep-ph/0606294] [INSPIRE]. 
[26] K. Cheung and T.-C. Yuan, Hidden fermion as milli-charged dark matter in Stueckelberg $Z^{\prime}$ model, JHEP 03 (2007) 120 [hep-ph/0701107] [INSPIRE].

[27] D. Feldman, Z. Liu and P. Nath, The Stueckelberg Z' Extension with Kinetic Mixing and Milli-Charged Dark Matter From the Hidden Sector, Phys. Rev. D 75 (2007) 115001 [hep-ph/0702123] [INSPIRE].

[28] D. Feldman, Z. Liu, P. Nath and B.D. Nelson, Explaining PAMELA and WMAP data through Coannihilations in Extended SUGRA with Collider Implications, Phys. Rev. D 80 (2009) 075001 [arXiv:0907.5392] [INSPIRE].

[29] ALEPH, DELPHI, L3, OPAL and SLD collaborations, LEP Electroweak Working Group, SLD Electroweak Group and SLD Heavy Flavour Group, Precision electroweak measurements on the $Z$ resonance, Phys. Rept. 427 (2006) 257 [hep-ex/0509008] [InSPIRE].

[30] Particle Data Group collaboration, Review of Particle Physics, Phys. Rev. D 98 (2018) 030001 [INSPIRE].

[31] C.-T. Huang, R. Ramos, V.Q. Tran, Y.-L.S. Tsai and T.-C. Yuan, Consistency of Gauged Two Higgs Doublet Model: Gauge Sector, JHEP 09 (2019) 048 [arXiv: 1905.02396] [INSPIRE].

[32] ATLAS collaboration, Search for high-mass dilepton resonances using $139 \mathrm{fb}^{-1}$ of $\mathrm{pp}$ collision data collected at $\sqrt{s}=13 \mathrm{TeV}$ with the ATLAS detector, ATLAS-CONF-2019-001.

[33] S. Davidson, S. Hannestad and G. Raffelt, Updated bounds on millicharged particles, JHEP 05 (2000) 003 [hep-ph/0001179] [InSPIRE].

[34] ATLAS collaboration, Search for dark matter and other new phenomena in events with an energetic jet and large missing transverse momentum using the ATLAS detector, JHEP 01 (2018) 126 [arXiv: 1711.03301] [INSPIRE].

[35] J.B. Muñoz and A. Loeb, A small amount of mini-charged dark matter could cool the baryons in the early Universe, Nature 557 (2018) 684 [arXiv: 1802.10094] [INSPIRE].

[36] K.K. Boddy, V. Gluscevic, V. Poulin, E.D. Kovetz, M. Kamionkowski and R. Barkana, Critical assessment of CMB limits on dark matter-baryon scattering: New treatment of the relative bulk velocity, Phys. Rev. D 98 (2018) 123506 [arXiv:1808.00001] [INSPIRE].

[37] R. de Putter, O. Doré, J. Gleyzes, D. Green and J. Meyers, Dark Matter Interactions, Helium and the Cosmic Microwave Background, Phys. Rev. Lett. 122 (2019) 041301 [arXiv: 1805.11616] [INSPIRE].

[38] E.D. Kovetz, V. Poulin, V. Gluscevic, K.K. Boddy, R. Barkana and M. Kamionkowski, Tighter limits on dark matter explanations of the anomalous EDGES $21 \mathrm{~cm}$ signal, Phys. Rev. D 98 (2018) 103529 [arXiv:1807.11482] [INSPIRE].

[39] J.M. Cline, G. Dupuis, Z. Liu and W. Xue, The windows for kinetically mixed Z'-mediated dark matter and the galactic center gamma ray excess, JHEP 08 (2014) 131 [arXiv:1405.7691] [INSPIRE].

[40] Planck collaboration, Planck 2015 results. XIII. Cosmological parameters, Astron. Astrophys. 594 (2016) A13 [arXiv:1502.01589] [INSPIRE].

[41] R. Foot, Implications of the DAMA and CRESST experiments for mirror matter type dark matter, Phys. Rev. D 69 (2004) 036001 [hep-ph/0308254] [INSPIRE]. 
[42] J.M. Cline, Z. Liu and W. Xue, Millicharged Atomic Dark Matter, Phys. Rev. D 85 (2012) 101302 [arXiv: 1201.4858 ] [INSPIRE].

[43] CMS collaboration, Search for new physics in final states with an energetic jet or a hadronically decaying $W$ or $Z$ boson and transverse momentum imbalance at $\sqrt{s}=13 \mathrm{TeV}$, Phys. Rev. D 97 (2018) 092005 [arXiv:1712.02345] [INSPIRE].

[44] J. Alwall et al., The automated computation of tree-level and next-to-leading order differential cross sections and their matching to parton shower simulations, JHEP 07 (2014) 079 [arXiv: 1405.0301] [INSPIRE].

[45] T. Sjöstrand et al., An Introduction to PYTHIA 8.2, Comput. Phys. Commun. 191 (2015) 159 [arXiv: 1410.3012] [INSPIRE].

[46] L. Carloni, J. Rathsman and T. Sjöstrand, Discerning Secluded Sector gauge structures, JHEP 04 (2011) 091 [arXiv: 1102.3795] [INSPIRE].

[47] B. Dumont et al., Toward a public analysis database for LHC new physics searches using MADANALYSIS 5, Eur. Phys. J. C 75 (2015) 56 [arXiv:1407.3278] [inSPIRE].

[48] D. Sengupta, The MadAnalysis5 implementation of the ATLAS in monojet+missing energy, DOI.

[49] CMS collaboration, Technical proposal for a mip timing detector in the cms experiment phase 2 upgrade, CERN-LHCC-2017-027.

[50] C. Allaire et al., Beam test measurements of Low Gain Avalanche Detector single pads and arrays for the ATLAS High Granularity Timing Detector, 2018 JINST 13 P06017 [arXiv: 1804.00622] [INSPIRE].

[51] ATLAS LAR-HGTD Group collaboration, A High-Granularity Timing Detector in ATLAS: Performance at the HL-LHC, Nucl. Instrum. Meth. A 924 (2019) 355 [InSPIRE].

[52] LHCb collaboration, Expression of Interest for a Phase-II LHCb Upgrade: Opportunities in flavour physics and beyond, in the HL-LHC era, CERN-LHCC-2017-003.

[53] J. Liu, Z. Liu and L.-T. Wang, Enhancing Long-Lived Particles Searches at the LHC with Precision Timing Information, Phys. Rev. Lett. 122 (2019) 131801 [arXiv:1805.05957] [INSPIRE].

[54] J.D. Mason, Time-Delayed Electrons from Higgs Decays to Right-Handed Neutrinos, JHEP 07 (2019) 089 [arXiv: 1905.07772] [INSPIRE].

[55] Z. Flowers, Q. Meier, C. Rogan, D.W. Kang and S.C. Park, Timing information at HL-LHC: Complete determination of masses of Dark Matter and Long lived particle, JHEP 03 (2020) 132 [arXiv: 1903.05825] [INSPIRE].

[56] O. Cerri, S. Xie, C. Pena and M. Spiropulu, Identification of Long-lived Charged Particles using Time-Of-Flight Systems at the Upgraded LHC detectors, JHEP 04 (2019) 037 [arXiv: 1807.05453] [INSPIRE].

[57] CMS collaboration, Measurement of charged pion, kaon and proton production in proton-proton collisions at $\sqrt{s}=13$ TeV, Phys. Rev. D 96 (2017) 112003 [arXiv: 1706.10194] [INSPIRE].

[58] S. Catani, M. Fontannaz, J.P. Guillet and E. Pilon, Cross-section of isolated prompt photons in hadron hadron collisions, JHEP 05 (2002) 028 [hep-ph/0204023] [INSPIRE]. 
[59] ATLAS collaboration, Measurement of the Inelastic Proton-Proton Cross section at $\sqrt{s}=13$ TeV with the ATLAS Detector at the LHC, Phys. Rev. Lett. 117 (2016) 182002 [arXiv: 1606.02625] [INSPIRE].

[60] A. Sopczak et al., Precision Luminosity of LHC Proton-Proton Collisions at 13 TeV Using Hit Counting With TPX Pixel Devices, IEEE Trans. Nucl. Sci. 64 (2017) 915 [arXiv: 1702.00711] [INSPIRE].

[61] CMS collaboration, Measurement and QCD analysis of double-differential inclusive jet cross sections in pp collisions at $\sqrt{s}=8 \mathrm{TeV}$ and cross section ratios to 2.76 and $7 \mathrm{TeV}$, JHEP 03 (2017) 156 [arXiv : 1609.05331] [INSPIRE].

[62] A. Alloul, N.D. Christensen, C. Degrande, C. Duhr and B. Fuks, FeynRules 2.0 - A complete toolbox for tree-level phenomenology, Comput. Phys. Commun. 185 (2014) 2250 [arXiv: 1310.1921] [INSPIRE].

[63] J. Chen, P. Ko, H.-N. Li, J. Li and H. Yokoya, Light dark matter showering under broken dark U(1) - revisited, JHEP 01 (2019) 141 [arXiv: 1807.00530] [INSPIRE].

[64] LHCb collaboration, Search for $A^{\prime} \rightarrow \mu^{+} \mu^{-}$Decays, Phys. Rev. Lett. 124 (2020) 041801 [arXiv: 1910.06926] [INSPIRE].

[65] LHCb collaboration, Measurements of prompt charm production cross-sections in pp collisions at $\sqrt{s}=13 \mathrm{TeV}$, JHEP 03 (2016) 159 [Erratum ibid. 09 (2016) 013] [Erratum ibid. 05 (2017) 074] [arXiv: 1510.01707] [InSPIRE].

[66] S. Benson, V.V. Gligorov, M.A. Vesterinen and M. Williams, The LHCb Turbo Stream, J. Phys. Conf. Ser. 664 (2015) 082004 [InSPIRE].

[67] P. Ilten, Y. Soreq, J. Thaler, M. Williams and W. Xue, Proposed Inclusive Dark Photon Search at LHCb, Phys. Rev. Lett. 116 (2016) 251803 [arXiv: 1603.08926] [INSPIRE].

[68] LHCb collaboration, Search for Dark Photons Produced in 13 TeV pp Collisions, Phys. Rev. Lett. 120 (2018) 061801 [arXiv:1710.02867] [INSPIRE].

[69] https://gitlab.com/philten/darkcast. 